\title{
Surgical resection of low-grade gliomas in eloquent areas with the guidance of the preoperative functional magnetic resonance imaging and craniometric points
}

\author{
Ahmed Abdullah, Hisham El Shitany, Waleed Abbass, Amr Safwat, Amr K Elsamman, Ehab El Refaee \\ Department of Neurosurgery, Cairo University, Cairo, Egypt
}

\begin{abstract}
Objectives: Surgical resection of low-grade gliomas (LGGs) in eloquent areas is one of the challenges in neurosurgery, using assistant tools to facilitate effective excision with minimal postoperative neurological deficits has been previously discussed (awake craniotomy and intraoperative cortical stimulation); however, these tools could have their own limitations thus implementation of a simple and effective technique that can guide to safe excision is needed in many situations. Materials and Methods: The authors conducted a retrospective analysis of a prospectively collected data of 76 consecutive surgical cases of LGGs of these 21 cases were situated in eloquent areas. Preoperative functional magnetic resonance imaging (fMRI), pre- and post-operative MRI with volumetric analysis of the tumor size was conducted, and intraoperative determination of the craniometric points related to the tumor (navigation guided in 10 cases) were studied to evaluate the effectiveness of the aforementioned tools in safe excision of the aforementioned tumors. Results: Total-near total excision in $14(66.67 \%)$ subtotal in $6(28.57 \%)$, and biopsy in 1 case $(4.57 \%)$. In long-term follow-up, only one case experienced persistent dysphasia. Conclusion: In spite of its simplicity, the identification of the safe anatomical landmarks guided by the preoperative fMRI is a useful technique that serves in safe excision of LGGs in eloquent areas. Such technique can replace intraoperative evoked potentials or the awake craniotomy in most of the cases. However, navigation-guided excision might be crucial in deeply seated and large tumors to allow safe and radical excision.
\end{abstract}

Key words: Craniometric points, eloquent areas, functional magnetic resonance imaging, glioma, low-grade glioma, surgery

\section{Introduction}

Gliomas are tumors arising in the cerebral parenchyma and have infiltrative growth pattern. ${ }^{[1]}$ Low-grade astrocytomas and oligodendrogliomas are types of gliomas that have slow growth and are associated with an apparently long natural history. ${ }^{[2]}$ They form about $20 \%$ of all central nervous system glial tumors. ${ }^{[3]}$ Due to their infiltrative pattern and aggressive behavior,

Address for correspondence:

Dr. Med. Ehab El Refaee, Department of Neurosurgery,

Cairo University, Cairo, Egypt.

E-mail: e.elrefaee@googlemail.com

\begin{tabular}{|l|l|}
\hline \multicolumn{2}{|c|}{ Access this article online } \\
\hline Quick Response Code: & Website: \\
\hline & www.ruralneuropractice.com \\
\cline { 2 - 2 } & \\
\hline
\end{tabular}

the mean 10 -year survival is $30 \%$ if $<90 \%$ of the tumor is resected. ${ }^{[3,4]}$ The ideal management of patients with low-grade glioma (LGG) is still controversial. This controversy mainly comes from the deficiency of well-designed clinical trials having an appropriate follow-up to run with the long progression-free survival and overall survival of patients. ${ }^{[5]}$ It is generally accepted that total or near total resection, may increase seizure-control and survival. Using measures such as intraoperative magnetic resonance imaging (MRI) may improve outcomes as compared to literature controls. ${ }^{[6,7]}$ Awake surgeries with intraoperative

This is an open access article distributed under the terms of the Creative Commons Attribution-NonCommercial-ShareAlike 3.0 License, which allows others to remix, tweak, and build upon the work non-commercially, as long as the author is credited and the new creations are licensed under the identical terms.

\section{For reprints contact: reprints@medknow.com}

How to cite this article: Abdullah A, El Shitany $H$, Abbass W, Safwat $A$, Elsamman AK, El Refaee E. Surgical resection of low-grade gliomas in eloquent areas with the guidance of the preoperative functional magnetic resonance imaging and craniometric points. J Neurosci Rural Pract 2016;7:571-6. 
mapping may also enhance functional outcomes and the quality of life..$^{[5,8]}$

Surgical treatment of LGGs aims to increasing the amount of tumor tissue excised, whereas lowering the risk of poor functional outcome. ${ }^{[9,10]}$ Neurosurgeons depended for a long time on standard functional maps to operate on brain lesions. ${ }^{[11]}$ With the revolution of functional neuroimaging, a vast amount of data demonstrated obvious interindividual variables in eloquent area location and the presence of plasticity phenomena. ${ }^{[12,13]}$ Variability between individuals, cortical plasticity, and distortion due to the mass effect make anatomical referencing insufficient. ${ }^{[12,14-16]}$ The integration of pre- and intra-operative brain mapping is an important progress in neurosurgery and allows the management of complex brain lesions while enhancing the quality of life. Yet, the use of preoperative mapping and its intraoperative efficiency is still debatable..$^{[15,17]} \mathrm{In}$ addition, the use of the aforementioned assistant tools is not available for all centers.

According to Ribas et al., there are sulcal and gyral key points that can be useful for intraoperative sulci identification. Together, they form a frame that may be helpful in the hemispheric lesion localization, in the placement of craniotomies, as landmarks for the approaches to periventricular lesions, and in anatomic guidance of removal of gyri that with infiltrative tumors. ${ }^{[18]}$

The objective of this study is to assess surgical and functional neurological outputs of LGG surgeries in eloquent areas using preoperative functional MRI (fMRI) with the guidance of the intraoperative anatomical landmarks, as an alternative to implementing awake craniotomy and intraoperative cortical brain mapping.

\section{Materials and Methods}

Seventy-six successive patients who underwent surgery for excision of LGGs during the years 2010-2014 in Cairo University Hospitals were collected from the Department of Neurosurgery database. Of these patients, 21 patients had the lesion in eloquent areas, performed a preoperative fMRI. The cortical areas of the language system were mapped with fMRI in all patients using a verb-generation task $(1.5 \mathrm{~T})$. In addition, the following was tested during the fMRI in all cases: Object naming, counting, and language differentiation.

Anatomical landmarks in relation to the tumor and in accordance with the preoperative fMRI were identified in the procedure. In ten patients, navigation-guided excision was performed due to their deeply seated nature.

The inclusion criteria included patients in adult age group, radiologically suspected LGG in close vicinity to eloquent areas, and deficit-free patients. The exclusion criteria included previously operated cases and lesions in noneloquent areas of the brain.

Patients were thoroughly examined pre- and post-operative and lesions sizes were measured volumetrically comparing the pre- and post-operative MRI imaging. The percentage of excision was estimated. The neurological deficits were stated as occurred or not as regard the motor, sensory, and speech systems.

\section{Demographic data}

Among the 21 patients, 9 patients were females (42.86\%), whereas males constituted $57.14 \%(n=12)$, the mean age was 42.85 (ranging from 28 to 62 years).

\section{Evaluation criteria}

In this study, we assessed the degree of benefit from using an integrated technique (discussed later) by evaluating the extent of excision in the follow-up MRI (of the mean of 6 months) and the postoperative neurological condition. Clinical condition, as well as pathological, radiological reports, and surgical data was prospectively documented. However, all this data were analyzed retrospectively.

\section{Extent of excision}

Comparative evaluation with volumetric analysis was performed for the pre- and post-operative MRI to determine the degree of excision in the 6 months follow-up MRI [Table 1].

\section{Surgical technique}

In all patients, nearby eloquent areas were identified in the fMRI to avoid their injury during the approach. The anterior Sylvian point, with relation to the Wernicke's, Broca's areas, was used as a landmark in lesions near the Sylvian fissure, the rolandic points were used as landmarks in lesions extending to the motor area. ${ }^{[18]}$

Once anatomical landmarks were identified, a safe approach through sulcal or cortical points was planned, guided by data obtained from fMRI considering the relations of the tumor to the eloquent areas, and thus to the sulcal key points with their relation to the important skull landmarks.

After the tumor was identified, microscopic "en bloc" tumor dissection was performed to avoid tumor residuals. 
Together with surgeon's experience, irrigation-suction technique was used to identify the tumor from glial tissue; no retraction was used in all cases.

Added value of functional magnetic resonance imaging fMRI served in the identification of the relation between the tumor and the eloquent areas, especially Broca's and Wernicke's areas. It was also beneficial preoperatively in decision-making by identification of the pattern of tumor growth. In cases where the tumor was infiltrating the eloquent areas; surgical excision was not advised, and only a stereotactic biopsy was performed. Identification of the double representation of speech areas was of value and served in radical excision. In one case, the left Broca's area was resected depending on the preoperative fMRI that indicated the dominancy of the right Broca's area. All cases included in this study were planned preoperatively for excision after studying all aforementioned factors through the fMRI.

Navigation-guided excision was used in ten patients due to their deeply seated position. Once a safe cortical window was settled, the tumor was removed en bloc with high magnification for better identification of the tumor from the glial tissue. We applied no retraction to the brain tissue in all cases. Bipolar cauterization was used with a very low current and as minimal as possible to coagulate tumor vessels.

\section{Results}

\section{Preoperative data}

Tumors were located in the dominant hemisphere in 13 cases $(61.9 \%)$, seven of these lesions extended in the frontal lobe, wherein six cases, the tumor was encroaching on the motor area. Five were extending in the dominant temporal lobe near - within Wernicke's area and in one case, the tumor was extending through the parietal lobe [Table 2].

\section{Clinical condition}

All patients had no neurological deficits preoperatively, six patients had only headache, ten with seizures, one with personality changes, two with numbness, and only one with attacks of dysphasia [Table 3].

\section{Clinical results}

In the immediate postoperative period, two cases had postoperative neurological deficits $(9.52 \%)$, one with weakness, and the other with dysphasia. In the long-term follow-up (more than 6 months), only one case had residual dysphasia (4.76\%). Otherwise, no other neurological deficit was identified [Table 4].

\section{Radiological results}

In the 6 months follow-up, among the 21 patients, five had a total resection (23.8\%), nine had near total (42.86\%), and six had a subtotal resection (28.57\%) [Figure 1]. Only one case was managed with excisional biopsy [Figure 2] as the tumor was infiltrating through the internal capsule [Table 5].

\section{Pathology}

Pathological examination revealed that most of the tumors were astrocytoma Grade II, except two cases who had the pathology of oligodendroglioma Grade II [Table 6].

Table 1: The extent of resection as expressed in a percentage from the original tumor size

\begin{tabular}{ll}
\hline Percentage excised $(\%)$ & Evaluation \\
\hline$<60$ & Excisional biopsy \\
$60-90$ & Subtotal \\
$90-100$ & Near total \\
100 & Total \\
\hline
\end{tabular}

Table 2: Site of lesions

\begin{tabular}{lc}
\hline Site & Number of patients (\%) \\
\hline Left frontal & $7(33.3)$ \\
Left temporal & $5(23.8)$ \\
On left motor area & $6(28.6)$ \\
Others & $8(38.1)$ \\
\hline
\end{tabular}

Table 3: Clinical presentation of cases

\begin{tabular}{lc}
\hline Presentation & Number of cases $(\%)$ \\
\hline Headache & $6(28.57)$ \\
Seizures & $10(47.62)$ \\
Personality changes & $1(4.76)$ \\
Numbness & $2(9.52)$ \\
Dysphasia (attacks) & $1(4.76)$ \\
\hline
\end{tabular}

Table 4: The immediate and 6 months follow-up results

\begin{tabular}{lc}
\hline Percentage of postoperative neurological deficits & $\%$ \\
\hline Immediate postoperative (\%) & 9.52 \\
6 months follow-up (\%) & 4.76 \\
\hline
\end{tabular}

Table 5: Postoperative extent of excision

\begin{tabular}{lc}
\hline Extent of resection & Number of cases (\%) \\
\hline Total & $5(23.8)$ \\
Subtotal & $6(28.57)$ \\
Near total & $9(42.86)$ \\
Biopsy & $1(4.76)$ \\
\hline
\end{tabular}

Table 6: Pathology of excised lesions

\begin{tabular}{lc}
\hline Pathology & Number of cases $(\%)$ \\
\hline Astrocytoma & $19(90.47)$ \\
Oligodendroglioma & $2(9.52)$ \\
\hline
\end{tabular}




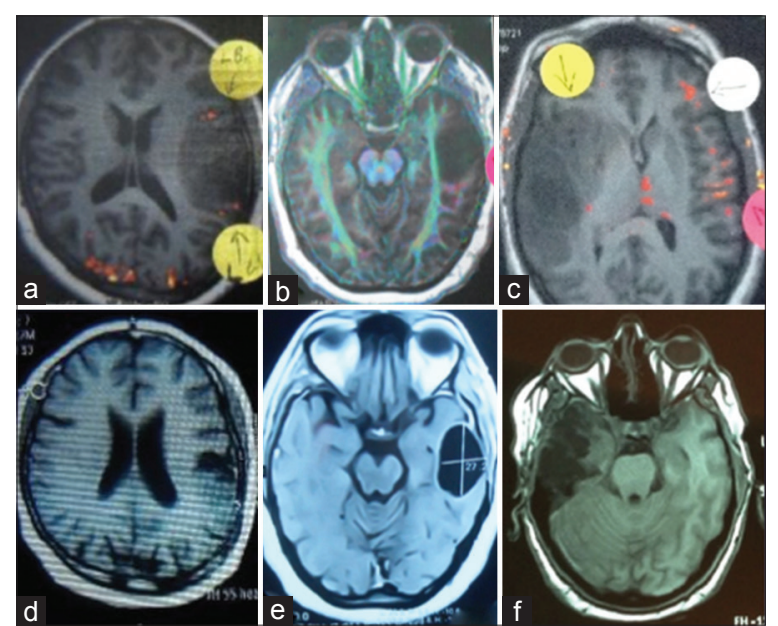

Figure 1: Preoperative functional magnetic resonance imaging "Axial T1 cuts" from three cases showing the relation of the tumor to the nearest eloquent area as left Broca's area (a) corticospinal tracts (b) and right Broca's area (c), where postoperative magnetic resonance imaging showing near total excision of the tumor in the three cases respectively (d-f)

\section{Discussion}

Resection of infiltrative lesions such as LGG located in eloquent brain areas is still a neurosurgical challenge. ${ }^{[19]}$ Although the modern neurosurgical practice is almost totally relying on the innovative neuronavigation technologies, the use of intraoperative craniocerebral relationships is still mandatory for the neurosurgeons, especially in centers with moderate-limited resources worldwide, and also for the young neurosurgeons who need a good understanding of the three dimensional anatomy of the brain and the cranial landmarks. ${ }^{[18]}$ This study aims at the integration of the transoperative determination of the cerebral landmarks and the preoperative fMRI to maximize the extent of resection and minimize the deficit in LGG cases, especially in centers with limited resources.

Awake surgery has been shown to improve the extent of resection for LGGs in eloquent areas. ${ }^{[8,16]}$ It is quite logical how the awake surgery plays a big role when gross total resection of tumor near an eloquent area is targeted, which would be a major predictor of outcome, aiming at keeping the functional neurological integrity, and better quality of life. ${ }^{[1]}$ However, awake surgery is not always the best option. Sometimes, it is too difficult for patients to tolerate the procedure that might be aborted intraoperatively due to patients anxiety. ${ }^{[20,21]}$

The risk-benefit balance of awake craniotomy depends also on risks associated with the patients' condition that may compromise surgery or even be life-threatening such as airway conformation and risk of obstructive

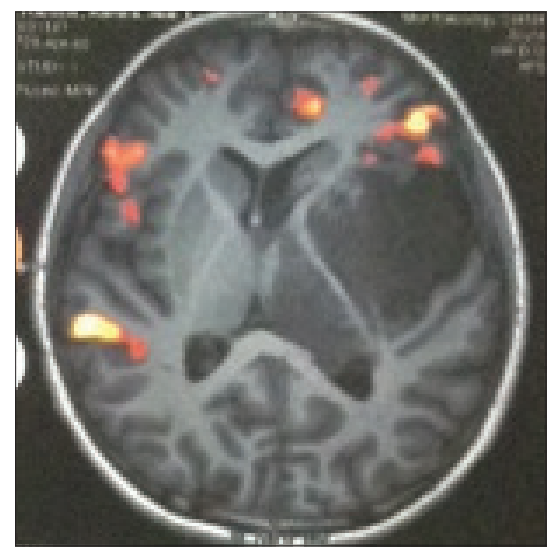

Figure 2: Functional magnetic resonance imaging (axial T1) showing infiltration of the internal capsule with the tumor. Excisional excisional biopsy was performed and revealed a low-grade glioma Grade II where the patient received radiotherapy

apnea, severity and frequency of seizures, propensity for nausea and vomiting, raised intracranial pressure, tendency for bleeding, patients' readiness to collaborate, anxiety and discomfort associated with the procedure, and presence of abnormal movements, and cognitive dysfunction that may render comprehension and collaboration difficult. fMRI helps in identification of language and motor centers. ${ }^{[2,22,23]}$ Those of sensory, motor, and language tasks are competently informative in patients with lesions near the eloquent cortex. Thus, fMRI is a reliable way of prediction of postoperative motor deficit risk following surgical excision. ${ }^{[23]}$ It also identifies more accurately the interindividual variations, increasing safety for surgery.

Intraoperative MRI may be potentially beneficial in increasing the surgical safety by providing real-time visualization of residual tumor and brain tissue. It also increases surgical accuracy by lowering neuronavigation errors due to intraoperative brain shift..$^{[5]}$ The intermixing between functional navigation and intraoperative MRI resulted in a less postoperative morbidity rates. ${ }^{[5,6]}$ However, many centers worldwide would have limited resources and development of a safe and simple approach to excise such lesions might be needed in many cases.

Awake craniotomy accompanied by cortical electrical stimulation helps in identification of the eloquent cortex, which cannot be reliably determined by anatomical landmarks. ${ }^{[24]}$ Motor evoked potentials in patients under general anesthesia have its own limiting factors. When recording complex muscle action potentials, the monitored muscles can only be controlled, so there would be potential inability to detect and even to avoid motor deficits in unmonitored group of muscles. ${ }^{[9,25]}$ In the extensive LGGs involving subcortical areas where 
the pyramidal tracts converge, all parts of the other half of the body may produce motor responses, making it difficult to find all muscle groups at risk before resection. Furthermore, "the motor evoked potentials would give only indirect information about the location of pyramidal pathway and do not give possibility for the identification of motor tracts at the resection interface ${ }^{\prime \prime} .{ }^{[9]}$ In addition, the use of the awake surgery or the intraoperative evoked potentials increases the operative time. In our technique, the use of the intraoperative anatomical landmarks guided with preoperative fMRI showed increases, respectively, the number of patients with same resected tumor $(66 \%$ of the total number of tumors operated upon with more than $90 \%$ resection of the tumor size), "total-near total" compared to the previous studies that found only (52\%) of the patients having a $>90 \%$ reduction in T2 signal postoperatively with awake craniotomy. ${ }^{[16]}$ The neurological deficit is $<9.52 \%$ in immediate postoperative assessment which decreased to $4.76 \%$ in the 6-month follow-up, which can decrease the fear of complications of tumor resection in eloquent areas whenever the access was determined craniometrically from a safe zone, en bloc dissection of the tumor with avoidance of major injuries to the surrounding tissue and guided with preoperative fMRI to detect the exact site of the eloquent areas at risk. The data provided by the preoperative fMRI, whether the tumor is invading or displacing the eloquent areas or tracts, served in better preoperative determination of the extent of resection and the risk of surgery.

In a former review of literature, reliability of the preoperative fMRI and the intraoperative cortical stimulation in surgeries near the speech areas was evaluated. Although their results concluded the accuracy of the fMRI, using it as a substitute to the cortical stimulation was not recommended. ${ }^{[16,23]}$

In our clinical study, we overcome the need to intraoperative cortical stimulation by identification of the site of the tumor to the nearest noneloquent cortex [Figure 1]. We implement this anatomical relationship technique with precise identification of the tumor to the skull and thus cortical landmarks, with acceptable long-term follow-up results.

Although using intraoperative navigation, fluorescence, MRI, cortical stimulation, and awake surgeries could be helpful in many cases. Safe and simple techniques would always be needed in several situations. We present this technique as a relatively safe and effective technique for excision of such tumors. However, a comparative study between such technique and awake surgeries is warranted.

\section{Conclusion}

In spite of its simplicity, the identification of the safe anatomical landmarks guided by the preoperative fMRI is a useful technique that serves in safe excision of LGGs in eloquent areas. Although less accuracy, such technique can be used in situations of the absence of intraoperative evoked potentials or the awake craniotomy. However, navigation-guided excision might be crucial in deeply seated and large tumors to allow safe and radical excision.

\section{Financial support and sponsorship \\ Nil.}

\section{Conflicts of interest}

There are no conflicts of interest.

\section{References}

1. Beez T, Boge K, Wager M, Whittle I, Fontaine D, Spena G, et al. Tolerance of awake surgery for glioma: A prospective European Low Grade Glioma Network multicenter study. Acta Neurochir (Wien) 2013;155:1301-8.

2. Bussell DS, Rubinstein LJ. Pathology of Tumours of the Nervous System. $4^{\text {th }}$ ed. London: Edward Arnold; 1977. p. 195-203.

3. Shields LB, Choucair AK. Management of low-grade gliomas: A review of patient-perceived quality of life and neurocognitive outcome. World Neurosurg 2014;82:e299-309.

4. Broca P. Instruction crainiologique et craniometrique de la Socite d'Anthropologie. Paris: G Masson; 1875.

5. Peruzzi P, Puente E, Bergese S, Chiocca EA. Intraoperative MRI (ioMRI) in the setting of awake craniotomies for supratentorial glioma resection. Acta Neurochir Suppl 2011;109:43-8.

6. Claus EB, Horlacher A, Hsu L, Schwartz RB, Dello-Iacono D, Talos F, et al. Survival rates in patients with low-grade glioma after intraoperative magnetic resonance image guidance. Cancer 2005;103:1227-33.

7. Chang EF, Clark A, Jensen RL, Bernstein M, Guha A, Carrabba G, et al. Multiinstitutional validation of the University of California at San Francisco low-grade glioma prognostic scoring system. Clinical article. J Neurosurg 2009;111:203-10.

8. De Benedictis A, Moritz-Gasser S, Duffau H. Awake mapping optimizes the extent of resection for low-grade gliomas in eloquent areas. Neurosurgery 2010;66:1074-84.

9. Duffau H, Capelle L, Denvil D, Sichez N, Gatignol P, Taillandier L, et al. Usefulness of intraoperative electrical subcortical mapping during surgery for low-grade gliomas located within eloquent brain regions: Functional results in a consecutive series of 103 patients. J Neurosurg 2003;98:764-78.

10. Yasargil MG, Krisht AF, Ture U, Al-Mefty O, Yasargil DC. Microneurosurgery of insular gliomas: Part II: Opening of the Sylvian fissure. Contemp Neurosurg 2002;24:1-5.

11. Spena G, D’Agata F, Panciani PP, Di Monale MB, Fontanella MM. Supratentorial gliomas in eloquent areas: Which parameters can predict functional outcome and extent of resection? PLoS One. 2013;8(12):1-8. doi:10.1371/journal.pone.0080916.

12. Hlustík P, Solodkin A, Noll DC, Small SL. Cortical plasticity during three-week motor skill learning. J Clin Neurophysiol 2004;21:180-91.

13. Bütefisch CM. Plasticity in the human cerebral cortex: Lessons from the normal brain and from stroke. Neuroscientist 2004;10:163-73.

14. Bello L, Gallucci M, Fava M, Carrabba G, Giussani C, Acerbi F, et al. Intraoperative subcortical language tract mapping guides surgical removal of gliomas involving speech areas. Neurosurgery 2007;60:67-80.

15. Berger MS, Ojemann GA. Intraoperative brain mapping techniques in neuro-oncology. Stereotact Funct Neurosurg 1992;58:153-61. 
16. Duffau H. Lessons from brain mapping in surgery for low-grade glioma: Insights into associations between tumour and brain plasticity. Lancet Neurol 2005;4:476-86.

17. Spena G, Nava A, Cassini F, Pepoli A, Bruno M, D’Agata F, et al. Preoperative and intraoperative brain mapping for the resection of eloquent-area tumors. A prospective analysis of methodology, correlation, and usefulness based on clinical outcomes. Acta Neurochir (Wien) 2010;152:1835-46.

18. Ribas GC, Yasuda A, Ribas EC, Nishikuni K, Rodrigues AJ Jr. Surgical anatomy of microneurosurgical sulcal key points. Neurosurgery 2006;59 4 Suppl 2:ONS177-210.

19. Duffau H, Capelle L, Denvil D, Sichez N, Gatignol P, Lopes M, et al. Functional recovery after surgical resection of low grade gliomas in eloquent brain: Hypothesis of brain compensation. J Neurol Neurosurg Psychiatry 2003;74:901-7.

20. Bonhomme V, Franssen C, Hans P. Awake craniotomy. Eur J Anaesthesiol 2009;26:906-12.

21. Otani N, Bjeljac M, Muroi C, Weniger D, Khan N, Wieser HG, et al. Awake surgery for glioma resection in eloquent areas - Zurich's experience and review. Neurol Med Chir (Tokyo) 2005;45:501-10.

22. Giussani C, Roux FE, Ojemann J, Sganzerla EP, Pirillo D, Papagno C. Is preoperative functional magnetic resonance imaging reliable for language areas mapping in brain tumor surgery? Review of language functional magnetic resonance imaging and direct cortical stimulation correlation studies. Neurosurgery 2010;66:113-20.

23. Pang BC, Wan WH, Lee CK, Khu KJ, Ng WH. The role of surgery in high-grade glioma - is surgical resection justified? A review of the current knowledge. Ann Acad Med Singapore 2007;36:358-63.

24. Meyer FB, Bates LM, Goerss SJ, Friedman JA, Windschitl WL, Duffy JR, et al. Awake craniotomy for aggressive resection of primary gliomas located in eloquent brain. Mayo Clin Proc 2001;76:677-87.

25. Berger MS, Deliganis A V, Dobbins J, Keles GE. The effect of extent of resection on recurrence in patients with low grade cerebral hemisphere gliomas. Cancer. 1994;74(6):1784-91. Available at: http://www.ncbi.nlm. nih.gov/pubmed/8082081. Accessed December 26, 2014. 